\title{
ANÁLISE DA EFICIÊNCIA ENERGÉTICA NO TRANSPORTE URBANO DE CURITIBA
}

\author{
L. P. Basile ${ }^{1}$ \\ ${ }^{1}$ Universidade Federal do Paraná, Centro Politécnico, Curitiba/PR, CEP 81531-970 \\ luisa.p.basile@gmail.com ${ }^{1}$
}

\begin{abstract}
Resumo: Em uma sociedade moderna, quase todas as atividades cotidianas acarretam custo de energia, razão pela qual a análise da eficiência energética se mostra importante, visto que busca otimizar o consumo desse insumo. Por sua vez, a porcentagem de energia útil em comparação ao total de energia consumida pelo setor de transportes no Brasil é de apenas 24,4\%, apresentando-se em desvantagem em relação aos setores industrial, residencial e comercial do país. Nesse contexto, o presente trabalho tem por objetivo analisar a eficiência energética de modais de transporte urbano de passageiros, utilizando-se do conceito de função produçáo. Para isso, foram utilizados dados de pesquisa realizada em corredores estruturais de transporte de Curitiba, contendo informaçóes sobre a frequência horária de diferentes modais de transporte e seus respectivos volumes de passageiros por hora, no período pico manhã. Por meio da aplicação de regressão múltipla e logarítmica nesses dados foi possível especificar dois modelos estatísticos, linear e não linear, relacionando, como variáveis independentes, as frequências de veículos de cada modal por hora com a variável dependente, volume total de viagens, em passageiros por hora. Dessa forma, foi possível estimar uma função produção para o uso de transporte na cidade de Curitiba. A partir da análise dessa função procurou-se relacionar seus coeficientes com a taxa média de ocupação de cada modal, de forma a estimar a sua produtividade média e estabelecer uma referência para análise da eficiência energética do transporte urbano de passageiros.
\end{abstract}

Palavras-chave: Eficiência energética, Transporte urbano, Função produção.

Abstract: In a modern society, almost all activities use energy, this is why the analysis of energy efficiency is important, since it seeks to optimize the consumption of that input. In this perspective, the percentage of useful energy compared to the total energy consumed by the transportation sector in Brazil is only $24.4 \%$, presenting a disadvantage compared to the industrial, residential and commercial sectors of the country. In this context, the present work aims to extend the understanding of urban transport energy efficiency and analyze it based on the concept of production function. In order to do so, we used survey data from Curitiba's transportation corridors, containing information on the hourly frequency of different modes of transport and their respective passenger volumes per hour in the morning peak period. By applying multiple and logarithmic regression in these data it was possible to specify two statistical models, linear and non-linear, relating, as independent variables, the vehicle frequencies of each modal per hour to the dependent variable, total travel volume, in passengers per hour. In this way, it was possible to estimate a production function for the use of transportation in the city of Curitiba. From the analysis of this function, it was possible to relate its coefficients with the average occupancy rate of each modal, in order to estimate its average productivity, establishing a reference for analysis of the urban transport energy efficiency.

Keywords: Energy efficiency, Urban transport, Production function. 


\section{Introduçáo}

O crescimento das cidades brasileiras de forma não sustentável vem causando diversos problemas ambientais, principalmente pela falta de planejamento da infraestrutura. Em 2000, mais de $80 \%$ da populaçáo morava em áreas urbanas, sendo que todas as atividades da cidade estão ligadas à necessidade de se deslocar (PONTES 1 , 2010 apud [1]). De acordo com [2] as redes de transporte, para apresentarem economias de escala, de escopo e de integridade necessitam ser adequadamente planejadas e desenvolvidas. Nesse sentido, e considerando-se que a mobilidade consome energia, ressalta-se a relevância do estudo sobre eficiência energética, que pode auxiliar na otimização desse consumo.

O presente trabalho, utilizando-se de uma base teórica, busca relacionar os conceitos e discussões sobre transporte urbano e seus modais públicos e particulares e a diferença de impacto ambiental e eficiência energética de cada um deles.

\subsection{Eficiência energética e economia do transporte urbano}

Uma discussão relevante em relação ao transporte urbano refere-se ao consumo energético decorrente dos diversos modais. Considerando que o transporte coletivo e o transporte individual têm praticamente a mesma participação modal, cada viagem do modal individual consome o triplo de energia do transporte coletivo (ANTP², 2014 apud [3]). De fato, "para cada passageiro de viagens de carro que se transferisse para o modo ônibus, haveria uma redução energética de 1,64 MJ/pass.km $[\text { sic }]^{3}$, o equivalente a $88 \%$ de economia da energia gasta pelo modo carro" $[3$, p. 6].

O conceito de economia do transporte, outra discussão relevante, é definido como "área aplicada da economia que está preocupada com o uso eficiente dos escassos recursos da sociedade para prover a movimentaçáo de pessoas e bens entre origem e um destino." [2]. Portanto, um sistema de transporte necessita ser planejado adequadamente, de forma a garantir tanto economia de escala, quanto integridade do sistema.

Para uma discussão sobre economia do transporte, o conceito de função produção se faz importante, ao relacionar os fatores de insumo e produtos. De acordo com [2] esse conceito representa as leis de proporção, descrevendo a transformação de insumos em produtos em um determinado período. Segundo [4], a função produção simbolicamente pode ser escrita como a Equação (1), Na qual $Y$, variável dependente, é o output ou quantidade de produto e $X_{1}, \ldots, X_{n}$, variáveis independentes, são diferentes inputs, ou insumos, que são usados no processo produtivo de $Y$.

\footnotetext{
${ }^{1}$ PONTES, T. F. Avaliação da mobilidade urbana na área metropolitana de Brasília. xxv, 250 p. Dissertação (Mestrado em Arquitetura e Urbanismo) - Faculdade de Arquitetura e Urbanismo, Universidade de Brasília, Brasília, 2010.

${ }^{2}$ ASSOCIAÇÃO NACIONAL DETRANSPORTES PÚBLICOS (ANTP). Sistema de informaçóes da mobilidade urbana: relatório geral 2012. São Paulo: ANTP, 2014. Disponível em: <http://antp.org.br/_5dotSystem/download/dcmDocument/2014/08/01/ CB06D67E-03DD-400E- 8B86-D64D78AFC553.pdf>. Acesso em: 15 dez. 2017.

${ }^{3} \mathrm{~A}$ forma correta de grafia para a unidade é $\mathrm{MJ} /$ passageiro $/ \mathrm{km}$. 


$$
Y=f \quad\left(X_{1}, X_{2}, X_{3}, \ldots, X_{n}\right)
$$

Essa função representa que para cada combinação de $X_{1}, \ldots, X_{n}$ existe apenas um nível de quantidade de produto $Y$. A função produção permite chegar a alguns conceitos úteis para a economia, tais como: produtividade marginal dos fatores de produção, elasticidade de substituição, fator intensidade, eficiência da produção, retornos de escala [2]. De acordo com esse autor, o produto marginal de um fator de produçáo representa como a mudança na produção de $Y$ varia com um incremento muito pequeno do fator variável $X_{1}, \ldots, X_{n}$, mantendo todos os outros constantes. De maneira matemática, o produto marginal, $M P$, de cada um dos fatores pode ser obtido pela derivada parcial da função produção em relação a esse fator, como na Equação (2), em que o fator considerado é $X_{1}$ :

$$
M P X 1=\frac{\partial Y}{\partial X 1}
$$

De forma gráfica o produto marginal de um fator $X_{1}$, mantendo as demais condiçóes constantes, significa a inclinação da função produção da Equação (3):

$$
Y=f \quad\left(X_{1}\right) x_{2}, x_{3}, \ldots, x_{n}
$$

Uma forma de obter-se uma função produção é utilizar-se da análise de regressão, que tem por finalidade descrever as relaçóes existentes entre duas ou mais variáveis, a partir de $n$ observaçóes dessas variáveis, por meio de um modelo estatístico. Uma opção é a regressão linear, a qual considera que a relação entre as variáveis é descrita por uma função linear. Quando é necessário analisar casos que tenham mais de uma variável independente - para o caso da função produção, mais de um fator de produção - é adequado utilizar-se da regressão múltipla. De acordo com [5, p. 4], "a análise de regressão linear múltipla é uma extensão da análise de regressão linear simples” e é aplicada quando há duas ou mais variáveis independentes para estimar o valor da variável dependente, formando assim a Equação (4) de regressão múltipla linear segundo [5]:

$$
y_{c}=a+b_{1} x_{1}+b_{2} x_{2} \ldots b_{k} x_{k}
$$

$\mathrm{Na}$ Equação 4, $y_{c}$ representa o "valor calculado de $y$ ", a é o intercepto-y, b, com respectivos índices, são coeficientes angulares, e $k$ é o número de variáveis independentes.

[5] também aponta três finalidades da regressão: estimarvalores de uma variável, com base em valores conhecidos das outras; explicar valores de uma variável em termos das outras; predizer valores futuros de uma variável, isto é, explicar o potencial futuro de outra variável com base em uma ou mais variáveis. 
Outra opção para se obter uma função produção é utilizar a regressão não linear, na qual a relação entre as variáveis não pode ser descrita por uma função linear, podendo ser escritas em forma de função exponencial ou logarítmica.

Uma análise importante a ser feita após a aplicação da regressão refere-se ao coeficiente de determinação (R-quadrado), o qual, segundo [5, p. 6], "indica a proporçâo da variância da variável dependente que pode ser estatisticamente atribuída ao conhecimento de uma ou mais variáveis independentes”. Assim, este coeficiente mensura a relação entre a variável dependente e as variáveis independentes, indicando quanto por cento a variação explicada pela regressão representa da variação total [5]. Quanto mais o R-quadrado é próximo de 1 , maior o grau de relacionamento entre as variáveis independentes e dependentes e quando R-quadrado é igual a um, de acordo com [5], na função especificada as variaçôes de $y$ (variável dependente) são 100\% explicadas pela variação dos $x x$ (variáveis independentes), sem desvios em torno da função estimada. Por sua vez, quando R-quadrado é igual a zero, pode-se dizer que as variaçôes de $y$ são exclusivamente aleatórias e as variáveis $x x$ não afetam as variaçôes de $y$.

\section{Metodologia}

Para o desenvolvimento da pesquisa foram utilizados dados obtidos de trabalhos domiciliares da disciplina TT057 Transporte Público, do Curso de Engenharia Civil, do Setor de Tecnologia da Universidade Federal do Paraná. Foram utilizadas informaçôes sobre a frequência dos modais automóvel, ônibus, motocicleta, bicicleta e (ônibus) biarticulado, por hora, no horário pico manhã (dados de corte transversal), em cinco corredores estruturais de transporte de Curitiba (Leste, Oeste, Sul, Norte e Boqueirão), divididos em vias lenta, rápida e canaleta de ônibus. Apenas nos casos dos corredores Leste e Boqueirão foi desconsiderada a separação em vias lenta e rápida e considerou-se a via adjacente à canaleta, já que o sistema conhecido como "trinário" " não está bem definido nesta região. Ressalta-se aqui, além da utilização de dados de corte transversal, outra restrição da pesquisa, decorrente dos poucos dados disponíveis sobre mais pontos da cidade. A Tabela 1 ilustra a planilha de dados de frequência.

Tabela 1 - Modelo de planilha de dados de frequência dos modais.

\begin{tabular}{|c|c|c|c|c|c|c|c|c|c|c|c|}
\hline \multirow{3}{*}{ Corredor } & \multirow{3}{*}{ Pico } & & & & \multicolumn{7}{|c|}{ Frequência (veículos/hora) } \\
\hline & & \multicolumn{2}{|c|}{ Canaleta } & \multicolumn{4}{|c|}{ Lenta } & \multicolumn{4}{|c|}{ Rápida } \\
\hline & & Biarticulado & Bicicleta & Automóvel & Ônibus & Moto & Bicicleta & Automóvel & Ônibus & Moto & Bicicleta \\
\hline Oeste & $7: 15-8: 14$ & 13 & 1 & 112 & 43 & 10 & 2 & 2.489 & 24 & 171 & 0 \\
\hline Norte & $7: 15-8: 14$ & 26 & 38 & 689 & 0 & 38 & 0 & 2.197 & 133 & 146 & 0 \\
\hline Sul & $7: 15-8: 14$ & 51 & 99 & 734 & 0 & 74 & 9 & 3.583 & 53 & 228 & 1 \\
\hline Boqueirão & $7: 30-8: 29$ & 23 & & 858 & 1 & & & & & & \\
\hline Leste & $7: 15-8: 14$ & 28 & & 1.487 & 28 & & & & & & \\
\hline
\end{tabular}

\footnotetext{
${ }^{4}$ O Sistema Trinário de Vias compreende via central, "canaleta central exclusiva para a circulação das linhas expressas (transporte de massa) e duas vias lentas para acesso às atividades lindeiras. A via exclusiva confere ganhos significativos para a velocidade operacional das linhas expressas", e vias estruturais, "duas vias paralelas à via central com sentido único, situadas a uma quadra de distância do eixo, destinadas às ligaçóes centro-bairro e bairro-centro, para a circulação dos veículos privados.”. [6]. 
Esta pesquisa se baseou também na taxa de ocupação média em cada um dos modais estudados, ilustrada na Tabela 2. Essa taxa, para ônibus biarticulados, foi obtida por meio de observaçáo visual em cada modal e corredor viário na hora pico manhã, sendo então somado para cada corredor viário o número de passageiros em cada biarticulado dividido pelo número de veículos biarticulados. As taxas de automóvel, ônibus, moto e bicicleta foram determinadas de forma análoga a taxa de biarticulados, mas por meio de amostragem, ou seja, não foram considerados todos os modais na hora pico manhã, então foi realizada uma expansão da amostra observada e média dos cinco pontos com valores aproximados.

Tabela 2 - Modelo de planilhas de taxa de ocupação média dos modais.

\begin{tabular}{ccc}
\hline \multicolumn{3}{c}{ Taxa média de ocupaçáo (passageiros/veículo) } \\
\hline & Oeste & 192,3 \\
Biarticulado & Norte & 177,3 \\
& Sul & 180,8 \\
& Boqueirão & 163,5 \\
Ônibus (genérico & Leste & 175,0 \\
Automóvel & & 60,0 \\
Moto & 1,5 \\
Bicicleta & 1,0 \\
\end{tabular}

Fonte: [7].

Dessa forma, multiplicando-se o número de carros pela taxa de ocupação média foi possível calcular o total de passageiros em cada modal, nos diferentes corredores viários. Por fim, por meio da soma desses números, foi determinado o total de frequências de passageiros por hora, em cada corredor, conforme ilustrado na Tabela 3.

Tabela 3 - Modelo de planilha contendo total de passageiros por modal.

\begin{tabular}{ccccc}
\hline Onibus & $\begin{array}{c}\text { Total por modo (passageiros/hora) } \\
\text { Automóvel }\end{array}$ & Moto & Bicicleta & $\begin{array}{c}\text { Total do corredor } \\
\text { (passageiros/hora) }\end{array}$ \\
\hline 6.520 & 3.902 & 181 & 3 & 10.605 \\
12.590 & 4.329 & 184 & 38 & 17.141 \\
12.401 & 6.476 & 302 & 109 & 19.287 \\
3.820 & 1.287 & & & 5.107 \\
6.580 & 2.231 & & & 8.811 \\
\hline
\end{tabular}

A partir dos dados organizados em planilha eletrônica, foi aplicada a regressão múltipla na própria planilha. Para isso, foram construídas duas novas tabelas: uma com os valores originais e outra com esses valores em escala logarítmica. Nessas tabelas, constavam apenas a frequência de cada modal 
e o total em cada corredor para o ônibus biarticulado, ônibus e automóveis, já que se optou por não considerar motocicleta e bicicleta, por suas taxas médias de ocupação serem unitárias.

Por meio da aplicação de regressão múltipla foi possível especificar uma equação linear, relacionando, como variáveis independentes, as frequências de veículos de cada modal por hora com a variável dependente, volume total de viagens, em passageiro por hora. Também foi realizada uma regressão múltipla logarítmica para se obter uma equação não linear.

\section{Resultados}

Utilizando-se dos dados presentes nas Tabelas 1 e 3 e aplicando-se a ferramenta regressão múltipla foi possível construir dois modelos estatísticos, um linear e outro não linear, que representam a função produção das viagens considerando os cinco corredores estruturais de transporte de Curitiba. Isso permitiu demonstrar como a variável produção de viagens varia à medida que o fator de produção de cada modal muda. Esse modelo considera para análise apenas o insumo capital de automóvel, biarticulado e ônibus. Dessa forma, a função produção, em sua forma genérica, seria como a Equação (5), na qual $Y$ representa o volume total de viagens, em passageiros por hora, e $X_{\text {automóvel }} X_{\text {biarticulado }}$ e $X$ ônibus o número de viagens por hora com automóvel, biarticulado e ônibus, respectivamente.

$$
Y=f \quad\left(X_{\text {automóvel }}, X_{\text {biarticulado }}, X_{\text {onibus }}\right)
$$

Para se chegar a um modelo mais preciso, foi necessária a comparação entre os resultados das regressôes múltipla linear e logarítmica. Para isso, analisou-se o valor de R-quadrado obtido nos dois modelos, concluindo-se que o modelo com valor de R-quadrado mais próximo de um e, portanto, que representaria de forma mais precisa o fenômeno estudado, seria o resultado da regressáo múltipla linear. Nesse contexto, nesta pesquisa não foi feita análise elaborada sobre o modelo e resultados da regressão logarítmica. Vale ressaltar que o valor de R-quadrado obtido é bastante elevado, aproximadamente 0,999 .

Observando o resultado da regressão linear e aproximando os valores, pode-se obter a Equação (6), que representa a função produção linear das viagens nos cinco corredores estudados.

$$
Y=182,8+166,9 X_{\text {biarticulado }}+58,7 X_{\text {ônibus }}+1,72 X_{\text {automóvel }}
$$

Após construção da equação partiu-se para a análise da produtividade marginal do modelo obtido. Considerando o produto marginal do fator de produção capital de cada modal como a derivada parcial da função produção em relação a esse fator, graficamente ele seria a inclinação da reta da função linear. Então, com a condição de que se mantenham constantes os demais fatores, caso a caso, a produtividade marginal de cada modal corresponderia ao coeficiente de cada uma das variá- 
veis independentes na equação obtida. Assim, a produtividade marginal, em passageiro(s) por hora, do automóvel seria aproximadamente 1,72; do ônibus seria 58,7 e do biarticulado seria 166,9.

Tomando-se estes coeficientes como uma taxa média de ocupação de cada modal, e tendo por base os dados disponibilizados por [8] e por [9] de consumo de combustível, e do padrão de conversão de combustível em energia adotado por [10], pode-se estimar a quantidade de energia consumida para transportar um passageiro por quilômetro para cada modal estudado. A Tabela 4 apresenta o consumo estimado de energia em MJ/passageiro/km de automóvel, biarticulado e ônibus convencional.

Tabela 4 - Consumo energético por passageiro/km por modo - Corredor Sul - Curitiba.

\begin{tabular}{cccc}
\hline Modo & Automóvel & Biarticulado & Ônibus convencional \\
\hline $\begin{array}{c}\text { Consumo Energético } \\
(\mathrm{MJ} / \text { passageiro } / \mathrm{km})\end{array}$ & 1,88 & 0,19 & 0,42 \\
\hline
\end{tabular}

Fonte: Elaborado pela autora.

Esses dados são úteis para comparar os níveis de eficiência energética entre os modais, que se mostram consideravelmente discrepantes. O automóvel utiliza mais de quatro vezes a quantidade de energia por passageiro/km utilizada pelo ônibus convencional e quase dez vezes a utilizada pelo ônibus biarticulado. Caso o passageiro do automóvel fosse para o modo biarticulado, a economia de energia seria de 1,69 MJ/passageiro/km, ou seja, uma redução de $90 \%$ do gasto energético do automóvel. Também vale ressaltar que a eficiência energética do biarticulado é aproximadamente o dobro da do ônibus convencional.

\section{Conclusóes}

A partir da pesquisa desenvolvida constata-se que a utilização da regressão linear permitiu obter um modelo estatístico capaz de representar o comportamento do uso de modais de transporte nas cinco regióes de Curitiba estudadas, em termos de passageiros transportados por hora. Entretanto, para que esse modelo seja mais preciso são necessárias informaçôes de mais pontos da cidade.

Foi possível também perceber, analisando-se os resultados dessa pesquisa e comparando-os com o estudo da Associação Nacional de Transportes Públicos (ANTP) (2014)5 disponibilizado por [3], que há uma diferença nos resultados em termos de consumo energético. Para o caso dos corredores estruturais de Curitiba, com a troca do modal individual para o transporte coletivo reduziu-se em mais de quatro vezes o consumo de energia e, para o caso do transporte coletivo biarticulado, em quase dez vezes. Também há uma diferença comparando com os resultados de [3], sendo a redução geral 2012. São Paulo: ANTP, 2014. Disponível em: <http://antp.org.br/_5dotSystem/download/dcmDocument/2014/08/01/ CB06D67E-03DD-400E- 8B86-D64D78AFC553.pdf>. Acesso em: 15 dez. 2017. 
energética resultante da mudança de automóvel para carro para este autor de 1,64 MJ/passageiro/km, enquanto nos dados obtidos nessa pesquisa foi de 1,69 MJ/passageiro $/ \mathrm{km}$.

Assim, percebe-se que sistemas estruturados de transporte coletivo, como o de Curitiba, permitem um ganho significativamente maior de eficiência energética em relação ao transporte individual. Por fim, os resultados da pesquisa apontam para a necessidade de políticas públicas de transporte urbano focadas no incentivo ao uso de transporte coletivo, ressaltando também a maior eficiência dos ônibus com mais capacidade, como o biarticulado, em relação ao ônibus convencional.

\section{Referências}

[1] LIMA, M. F. Externalidades do transporte e a mobilidade urbana do Distrito Federal. 75 p. Monografia (Bacharelado em Ciências Econômicas). Faculdade de Economia, Administração, Contabilidade e Ciência da Informação e Documentação, Universidade de Brasília, Brasília, 2014.

[2] SENNA, L. A. dos S. Economia e planejamento dos transportes. Rio de Janeiro: Elsevier, 2014.

[3] SILVA, L. V.; RECK, G. Eficiência energética e divisão modal no transporte urbano. S.d. Disponível em: <files.server.antp.org.br/_5dotSystem/download/dcmDocument/2015/06/15/C7D79AB7-8A43-45359E4B60B23C4672C1.pdf>. Acesso em: 15 dez. 2017.

[4] DOLL, J. P.; ORAZEM, F. Teoria da produçáo. Traduzido e adaptado, por J. C. S. M. dos Santos, de Production economics: theory with applications. New York: John Wiley \& Sons, 1984. Ponte de Lima: Escola Superior Agrária de Ponte de Lima, 2006.

[5] OLIVEIRA FILHO, M. L. A utilização da regressão linear como ferramenta estratégica para a projeção dos custos produção. In: IX CONGRESSO BRASILEIRA DE CUSTOS. Anais... São Paulo, 13 a 15 de outubro de 2002. Disponível em: <https://anaiscbc.emnuvens.com.br/anais/article/viewFile/2762/2762>. Acesso em: 15 dez. 2017.

[6] URBANIZAÇÃO DE CURITIBA S.A. (URBS). Sistema trinário de vias. Disponível em: <https://www.urbs. curitiba.pr.gov.br/transporte/rede-integrada-de-transporte/19>. Acesso em: 29 nov. 2017.

[7] SCHEREMETA, F. H. P. Análise da eficiência energética de veículos de transporte urbano. Curitiba: Universidade Federal do Paraná, 2017. Relatório de Iniciação Científica.

[8] URBANIZAÇÃO DE CURITIBA S.A. (URBS). Metodologia tarifária do Sistema Integrado de Transporte da Grande Curitiba. Curitiba: URBS, 2009. Disponível em: <http://www.urbs.curitiba.pr.gov.br/PORTAL/rit/ arquivosPDF/resumo_metodo_2009_site.pdf>. Acesso em: 15 dez. 2017.

[9] INSTITUTO NACIONAL DE METROLOGIA, QUALIDADE E TECNOLOGIA (Inmetro). Tabelas de consumo / eficiência energética. 2014. Disponível em: <http://www.inmetro.gov.br/consumidor/pbe/veiculos_ leves_2014.pdf>. Acesso em: 15 dez. 2017.

[10] INTERNATIONAL ENERGY AGENCY (IEA). Energy statistics manual. Paris: IEA, 2005. Disponível em: <http://www.iea.org/publications/freepublications/publication/energy-statistics_manual.pdf>. Acesso em: 15 dez. 2017. 